\title{
El Laboratorio Nacional de Referencia y su contribución a la eliminación de la tuberculosis en Cuba
}

\author{
The National Laboratory of Reference and its contribution \\ to the elimination of tuberculosis in Cuba
}

\author{
DrC. Ernesto Montoro Cardoso, ${ }^{\mathrm{I}}$ DrC. Raúl Díaz Rodríguez, ${ }^{\mathrm{II}}$ DrC. Dihadenys \\ Lemus Molina, ${ }^{\text {II }}$ MsC. Lilian Mederos Cuervo, ${ }^{\text {II }}$ MsC. Iliana Valdés \\ Hernández, II MsC. María Rosarys Martínez Romero, ${ }^{\text {II }}$ DraC. Alina Llop \\ Hernández, ${ }^{\mathrm{II}}$ DrCs. José A. Valdivia Álvarez ${ }^{\mathrm{II}}$ \\ I Laboratorio Nacional de Referencia e Investigaciones en Tuberculosis y Micobacterias. \\ Centro Colaborador OPS/OMS. Instituto de Medicina Tropical "Pedro Kourí" (IPK). \\ La Habana, Cuba.

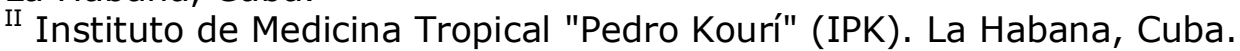

\section{RESUMEN}

Se describe la participación del Laboratorio Nacional de Referencia e Investigaciones en Tuberculosis y Micobacterias del Instituto de Medicina Tropical "Pedro Kourí", en la organización del recurso bacteriológico para el diagnóstico y la búsqueda de casos, fundamentalmente en la atención primaria de salud, en apoyo al Programa Nacional de Control de la Tuberculosis. Se hace referencia a las funciones y principales actividades científicas de este Laboratorio Nacional, Centro Colaborador OPS/OMS, como cabecera de los laboratorios de la red de diagnóstico de la tuberculosis en el país. La introducción y aplicación de nuevas tecnologías de avanzada permite contar con valiosas herramientas para dar respuesta a los diferentes retos que plantean la tuberculosis y las micobacteriosis en la actualidad. Con la ejecución de un proyecto del fondo mundial de lucha contra el sida, la tuberculosis y la malaria, se ha fortalecido la red de diagnóstico, lo que debe contribuir, en un futuro cercano, a la eliminación de la tuberculosis como problema de salud en Cuba.

Palabras clave: Tuberculosis, métodos diagnósticos, Laboratorio Nacional de Referencia, Centro Colaborador, red de laboratorios. 


\begin{abstract}
The participation of the National Laboratory of Reference and Research on Tuberculosis and Mycobacteria of "Pedro Kouri" Institute of Tropical Medicine in the organization of the bacteriological resources for the diagnosis and screening of cases in the primary health care, in support of the national program for control of tuberculosis, was described in this paper. Reference was made to the functions and main scientific activities of this laboratory, which is a PAHO/WHO collaborating center at the forefront of the tuberculosis diagnosis laboratory network in the country. The introduction and the implementation of state-of-the-art technologies have allowed valuable tools to be used to meet several challenges posed by tuberculosis and mycobacteria nowadays. Thanks to the implementation of a World Fund against AIDS, tuberculosis and malaria project, the diagnosis network has strengthened, which must contribute to the elimination of tuberculosis as a health problem in Cuba in the near future.
\end{abstract}

Key words: Tuberculosis, diagnostic methods, National Laboratory of Reference, Collaborating Center, laboratory network.

\title{
INTRODUCCIÓN
}

El diagnóstico, uno de los objetivos fundamentales del Programa Nacional de Control de Tuberculosis (PNCT), se basa en el examen microscópico directo del esputo para identificar los casos de tuberculosis (TB) con baciloscopia (BK) positiva o casos infecciosos, cuya detección es uno de los objetivos principales del programa.

Los servicios de bacteriología de la TB deben coordinar estrechamente sus actividades con los componentes administrativos, epidemiológicos y clínicos del PNCT. El diagnóstico bacteriológico se debe realizar conjuntamente con otros componentes del programa, para lograr la mayor cobertura e integrar las actividades en la estructura de los servicios generales de salud de un país o una región.

La red de laboratorios es, por lo tanto, una herramienta importante del diagnóstico bacteriológico de la TB. Esta red consiste en una estructura en la cual varios laboratorios, que trabajan a diferentes niveles de complejidad de servicio, se encuentran vinculados por los objetivos comunes del PNCT. La red es necesaria porque la BK, elemento esencial para el diagnóstico, debe llevarse a cabo en conformidad con las normas, con garantía de calidad y lo más cerca posible al domicilio del paciente. A su vez, la red suministrará la información requerida para planificar, vigilar y evaluar las actividades del programa a todos los niveles. ${ }^{1}$

\section{LABORATORIO NACIONAL DE REFERENCIA E INVESTIGACIONES EN TUBERCULOSIS Y MICOBACTERIAS}

Desde 1964 se norman en Cuba los métodos para el diagnóstico de la TB. A partir de 1969, con las nuevas modificaciones realizadas al Programa Nacional de Control,

http://scielo.sld.cu 
el Laboratorio Nacional de Referencia e Investigaciones en Tuberculosis y Micobacterias (LNR-TB), participa en la organización del recurso bacteriológico para la búsqueda de casos en apoyo a dicho programa. ${ }^{2}$

El LNR-TB del Instituto de Medicina Tropical "Pedro Kourí" (IPK), tiene como función principal la de ser centro de referencia nacional para la TB y otras micobacterias. Este laboratorio funge como cabecera de la red de laboratorios en el país, que comprende 13 laboratorios provinciales, 46 municipales, 138 en hospitales y 444 en policlínicos. En estos últimos laboratorios se realiza la BK para la búsqueda de casos en la atención primaria. En la actualidad, Cuba cuenta con un laboratorio para realizar BK por cada 18488 habitantes (la OMS recomienda un laboratorio por cada $10^{5}$ habitantes), y un laboratorio para el diagnóstico por cultivo por cada 229490 habitantes (se recomienda un laboratorio por cada $10^{6}$ habitantes).

EI LNR-TB desempeña otras funciones como son la normación, asesoría, supervisión, capacitación y control de la calidad. Debe además recibir cepas de micobacterias aisladas en los laboratorios de la red para su clasificación, y poder asimilar, de esta forma, los servicios técnicos especializados que el resto de los laboratorios no realizan. Es el encargado de planificar, en coordinación con el PNCT y en colaboración con los laboratorios de la red, los programas de adiestramientos y cursos de posgrado para el personal técnico y profesional, así como un programa de investigaciones sobre la base del problema y el desafío que plantea la TB en la actualidad. $^{2}$

En 1998 el LNR-TB fue designado Centro Colaborador OPS/OMS para la Referencia e Investigaciones en Tuberculosis y otras Micobacterias. Este Centro tiene entre sus términos de referencia participar en el programa internacional para la vigilancia de la resistencia patrocinada por la OMS, actuar como laboratorio de referencia en la región para el control de la calidad de la $\mathrm{BK}$, los cultivos y las pruebas de sensibilidad; desarrollar cursos de adiestramiento, participar en investigaciones internacionales mediante la ejecución de proyectos y desarrollar sistemas de información para el registro de los resultados del laboratorio.

Para dar cumplimiento a los términos de referencia, el Centro Colaborador en los últimos 10 años ha participado en los cuatro estudios mundiales para la vigilancia de la resistencia a las drogas antituberculosas de primera línea. Ha ejecutado proyectos de investigación con financiamiento de la Unión Europea, de la Agencia Iberoamericana de Ciencia y Técnica (CYTED) y del Consejo Nacional de Ciencia y Tecnología (CONACYT) de México. Ha trabajado en colaboración con el Instituto de Medicina Tropical de Amberes en Bélgica y con la Universidad de Murcia en España. Los resultados obtenidos en estos proyectos, ha permitido la obtención de grados científicos de los miembros del Centro Colaborador (tres doctores en ciencias, tres másteres en bacteriología-micología). Además, se han publicado varios artículos científicos en revistas internacionales de alto impacto.

Cada año el Centro Colaborador realiza un curso internacional de tuberculosis. Han participado hasta la fecha profesionales de Argentina, Brasil, Ecuador, España, Guatemala, México, Perú, República Dominicana y Venezuela, entre otros.

Investigadores de Brasil, Bolivia, Colombia, Estados Unidos, México, Panamá, Perú, República Dominicana, Suecia y Venezuela han realizado estancias científicas en el Centro Colaborador. Paralelamente, se ha brindado asesoría a la red de laboratorios de Brasil, Guatemala, Nicaragua, Perú y República Dominicana. 


\section{MÉTODOS PARA EL DIAGNÓSTICO DE LA TB}

\section{Baciloscopia}

La BK se realiza en todos los laboratorios de la red y en los policlínicos, lo cual brinda una cobertura total a todas las regiones del país. Esta técnica es sencilla, rápida y barata; constituye la piedra angular del diagnóstico de la TB y permite detectar a las personas enfermas capaces de transmitir la infección en la comunidad. Por otra parte, permite hacer el seguimiento del tratamiento y evaluar las tasas de curación. ${ }^{1}$

El control de la calidad de la BK, es esencial para el adecuado funcionamiento del laboratorio; está diseñado para mejorar de forma continua la confiabilidad y eficiencia de la BK, por lo que asegura que la información generada por los laboratorios sea exacta y confiable. ${ }^{3}$ EI PNCT establece la realización del control de la calidad a la BK, al $100 \%$ de las BK positivas y al $10 \%$ de las negativas, que se envían mensualmente desde los laboratorios de la red hacia los laboratorios de referencia provinciales (localizados en los Centros Provincial de Higiene, Epidemiología y Microbiología). A su vez, el LNR-TB controla a estos laboratorios siguiendo el mismo procedimiento. ${ }^{3}$

Recientemente, como parte de la ejecución de un proyecto internacional, el LNR-TB evaluó dos nuevos métodos para el control de la calidad de la BK: 1. el rechequeo de láminas a ciegas (RLC), con previa recoloración de las láminas antes de ser evaluadas y 2. El panel de láminas. En esta investigación participaron laboratorios seleccionados de las provincias de Ciudad de La Habana (actualmente La Habana) y Las Tunas. Las ventajas de estos métodos respecto al sistema clásico de rechequeo son: en el caso del RLC, se realiza la recoloración de las láminas antes de la relectura, lo que permite estimar con mayor precisión el comportamiento de la tasa de falsos positivos y falsos negativos. Además, el tipo de muestreo que utiliza esta nueva modalidad está diseñado para tomar el número más bajo de frotis que pueda indicar si un laboratorio alcanza un nivel mínimo de calidad predeterminado. De esta manera, el número de láminas a evaluar sería mucho menor, lo que evita sobrecargar de trabajo al personal del laboratorio que además, realiza el trabajo de diagnóstico. Otra ventaja es que la evaluación de las láminas se realiza a doble ciego y que existe otro laboratorio controlador con personal entrenado y capacitado. La desventaja radica en que este tipo de muestreo está diseñado para países con tasa de frotis positivos por encima del $5 \%$, por debajo de esta cifra se produce un sesgo a favor de las BK negativas y no se estimaría con exactitud el comportamiento de la tasa de falsos negativos. ${ }^{4,5}$

El panel de láminas se considera menos eficiente que el RLC. Este método no constituye un vigilante del desempeño de rutina del laboratorio, pero es de gran utilidad para suplementar los programas de rechequeo, provee datos preliminares acerca de las capacidades de los técnicos de laboratorio antes de implementar el RLC y ayuda a identificar problemas asociados con un pobre desempeño, lo que permite que sirva de sostén para evaluarlos, una vez que han recibido reentrenamiento. ${ }^{4,5}$

\section{Cultivo}

El examen por cultivo se realiza a nivel de los Centros de Higiene, Epidemiología y Microbiología provinciales y en 30 centros municipales, así como en los laboratorios 
de los principales hospitales. Este método constituye la prueba de oro para el diagnóstico definitivo de la TB. Mediante el cultivo, es posible aumentar la confirmación del diagnóstico de la enfermedad del 15-20\% del total de casos y del 20-30 \% de los pacientes con TB pulmonar. Además, detecta a los enfermos de forma temprana, antes de volverse infecciosos. Un programa de control de la calidad del cultivo, implementado debidamente, mejora de forma continua el rendimiento y eficiencia del cultivo, evalúa la calidad de los medios de cultivo en los laboratorios donde se realizan, detecta los errores sistemáticos y sostenidos en el tiempo a través del cálculo de los indicadores de la calidad de forma periódica, todo lo cual permite identificar las posibles causas que inciden y tomar medidas correctivas oportunas. ${ }^{6}$ Este programa se aplicó como parte de una investigación en las provincias de Ciudad de La Habana y Las Tunas.

\section{Cultivo automatizado}

En las últimas décadas se han desarrollado diferentes métodos automatizados para el cultivo de micobacterias. Dentro de ellos tenemos el Sistema Radiométrico BACTEC-460®TB, MGIT (Mycobacterium growth indicador tube), Versa TREK, Bact/ALERT 3D, entre otros. ${ }^{7}$

En estos momentos el LNRTB- IPK, dispone del equipo Bact/ALERT 3D para el diagnóstico rápido de micobacterias. Este sistema presenta una alta sensibilidad y especificidad, es un sistema no invasivo, permite la monitorización y detección rápida, de forma sensible y confiable de las micobacterias en esputo, sangre y otros líquidos corporales. El tiempo de detección del crecimiento para M. tuberculosis oscila entre 11 y 13 días como promedio y para las micobacterias de crecimiento rápido, de 2-3 días. El fundamento del equipo se basa en la presencia del sensor colorimétrico LES (Liquid Emulsion Sensor) de alta sensibilidad y la reflexión de la luz para monitorear la presencia y producción de $\mathrm{CO}_{2}$ disuelto en el medio de cultivo, derivado del metabolismo de los microorganismos. Una vez que los microorganismos producen $\mathrm{CO}_{2}$, el sensor líquido permeable al gas cambia de color de azul/verdoso a un amarillo, que se refleja en las unidades de reflactancia y en la gráfica que construye el sistema..$^{8,9}$

Dentro de las ventajas del equipo se encuentran: que el sistema es completamente automatizado, reduce costos por aumento de productividad, el monitoreo de las botellas no es invasivo, elimina el riesgo de contaminación cruzada y permite detectar los casos positivos de forma más rápida, el volumen de muestra es flexible, mide objetivamente el crecimiento microbiano, identifica de forma inmediata las muestras positivas por combinación de alarmas audibles y visuales, la identificación se realiza por código de barras lo que permite la trazabilidad de las muestras, permite cargar, descargar y localizar fácilmente las botellas en el sistema. ${ }^{8,9}$

La presencia de este instrumento en el LNRTB-IPK, es una herramienta útil porque permitirá realizar el diagnóstico de TB en un menor tiempo, se dispone de una tecnología de punta que permitirá fortalecer el diagnóstico bacteriológico de la TB, sobre todo en los pacientes VIH, que ocupan más del $70 \%$ de los casos que se estudian en el IPK y generalmente son negativos a la BK al reducir el tiempo de diagnóstico y establecer un tratamiento precoz y oportuno de la enfermedad, además, a partir de la botellas positivas a M. tuberculosis se podrán realizar de forma directa las pruebas de susceptibilidad a las drogas antituberculosas. 


\section{Identificación de micobacterias del complejo Mycobacterium tuberculosis}

Se realiza a través de las pruebas bioquímicas convencionales de niacina, termoestabilidad de la enzima catalasa a $68^{\circ} \mathrm{C}$ y la reducción de nitrato; estas pruebas tienen el inconveniente de ser muy laboriosas, además de utilizar reactivos químicos tóxicos. ${ }^{6}$

En los últimos años se han desarrollado nuevas tecnologías encaminadas a acelerar y simplificar la detección del complejo M. tuberculosis a partir del crecimiento en medios sólidos o líquidos. Actualmente, varias casas comerciales disponen del test para la identificación rápida por ensayo de inmunocromatografía que utilizan anticuerpos monoclonales contra el antígeno (Ag) MPT64, presente solamente en este complejo. Es fácil de realizar, con una sensibilidad del $99 \%$ y especificidad del $100 \%$, no requiere de personal altamente calificado ni de equipamiento sofisticado para realizar la prueba. La prueba positiva al antígeno se evidencia por la aparición de dos bandas color fucsia (banda control y la banda de la muestra que se está testando) ${ }^{10,11}$ Esta técnica se está utilizando en los laboratorios de la red que realizan cultivo.

\section{Susceptibilidad de Mycobacterium tuberculosis a las drogas antibacilares de primera línea}

Desde hace más de 50 años se dispone de la quimioterapia antituberculosa con drogas altamente eficaces. El tratamiento con las drogas antibacilares de primera línea es la acción más importante en los PNCT modernos. Tanto para su implantación como para su ulterior desarrollo, es de importancia básica el conocimiento de la resistencia existente en un país o región, lo que permite seleccionar los mejores esquemas, evaluar su eficacia, controlar el grupo crónico de pacientes, así como elegir el esquema de drogas más adecuado en los retratamientos. ${ }^{12}$

En la actualidad, la circulación de cepas multidrogorresistentes (MDR), resistentes al menos a la isoniacida (INH) y la rifampicina (RIF), las dos drogas más importantes del tratamiento y la reciente aparición de las cepas extremadamente resistentes (XDR), cepas MDR con resistencia añadida a alguna fluoroquinolona y a una de las tres drogas inyectables de segunda línea (amikacina, kanamicina y capreomicina), vuelven aún más difícil y costoso el control de la TB, lo que pudiera convertirse en una enfermedad incurable. ${ }^{13}$

Desde 1963, se describió el método de las proporciones, como la prueba de referencia para los estudios de susceptibilidad a las drogas antituberculosas. En Cuba, Valdivia y Suárez Méndez realizaron los primeros estudios en $1965,{ }^{14}$ y desde 1975 se ejecuta un estudio longitudinal de vigilancia de la resistencia de M. tuberculosis. ${ }^{15}$

En 1994, el Programa Mundial de TB de la OMS y la Unión Internacional contra la Tuberculosis y Enfermedades Respiratorias (UNION), iniciaron un proyecto mundial para la vigilancia de la resistencia a las drogas antituberculosas, que permitió en ese momento estimar la existencia de alrededor de 50 millones de personas infectadas con cepas MDR y más de 400000 casos nuevos de TB-MDR. ${ }^{16}$ 
Hasta la fecha se han realizado 4 estudios mundiales (1997, 2000, 2004 y 2008). Cuba es el único país de Iberoamérica que ha participado en todos los estudios. Los resultados obtenidos han permitido conocer la tendencia descendente de la resistencia en Cuba, lo que se corresponde con las altas tasas de curación de pacientes y bajas tasas de fracaso y abandono del tratamiento. Además, se ha demostrado la escasa circulación de cepas de M. tuberculosis MDR, con las cifras más baja en la región de Las Américas, lo cual difiere bastante de la situación mundial actual. ${ }^{16-20}$

Previo a la participación en cada uno de los estudios mundiales, el LNRTB-IPK se sometió a controles de calidad externo por parte de los Laboratorios de Referencia Supranacionales. En el último control realizado en el año 2007, correspondiente al cuarto estudio mundial, se obtuvo una sensibilidad y especificidad de $100 \%$ para las cuatro drogas evaluadas.

\section{Métodos rápidos para la detección de resistencia en Mycobacterium tuberculosis}

Entre ellos, el ya citado método de las proporciones, que ofrece resultados reproducibles y de elevada correlación clínica, sin embargo, tiene el inconveniente de requerir entre 4-6 semanas de incubación para obtener los resultados debido al crecimiento lento de $M$. tuberculosis. Esta limitante unida al impacto que tiene en nuestros días el fenómeno de la MDR ha hecho que la estandarización de técnicas rápidas para la detección de resistencia sea una de las líneas de investigación priorizadas que realiza el LNR-TB del IPK.

La evaluación de métodos alternativos para la detección de resistencia en el LNR-TB, le ha permitido incorporar al trabajo cotidiano el método de nitrato reductasa y adoptar un nuevo algoritmo para realizar un diagnóstico rápido de resistencia en cepas de M. tuberculosis. Recientemente, se ha comenzado a estudiar la sensibilidad a los fármacos antituberculosos de segunda línea para la detección de cepas extremadamente resistentes (XDR), así como el empleo de métodos moleculares (GenotypeMTBDRplus y GenotypeMTBDRs/) para detectar las mutaciones en los genes involucrados en la resistencia a fármacos de primera y segunda línea. Hasta el presente, no se han detectado cepas XDR en Cuba.

En la tabla se expone los métodos ensayados y se describe para cada uno de ellos las drogas empleadas y la precisión que ofrecen estas nuevas herramientas, en relación con la técnica de referencia utilizada en cada estudio. ${ }^{21-25}$

De los métodos evaluados, el de la nitrato reductasa (MNR) es fácil de realizar, se obtienen altos valores de concordancia y solo requiere para su ejecución del equipamiento básico del laboratorio. Consideramos que este método puede ser implementado en países de bajos recursos económicos donde la TB-MDR constituye un serio problema de salud. En la actualidad, se dispone del MNR para ser utilizado directamente en muestras de esputo con BK positiva, lo que reduce aún más el tiempo de obtención de resultados. 
Tabla. Resultados de los métodos rápidos para detección de resistencia de Mycobacterium tuberculosis a las drogas de primera línea

\begin{tabular}{|c|c|c|c|c|c|c|c|c|c|}
\hline \multirow{2}{*}{ Autor. Año } & \multicolumn{2}{|c|}{ Método / Tiempo } & \multirow{2}{*}{\begin{tabular}{|c|} 
No. \\
Cepas
\end{tabular}} & \multirow{2}{*}{ Drogas } & \multicolumn{5}{|c|}{ Concordancia (\%) } \\
\hline & Evaluado & Referencia & & & I & $\mathrm{S}$ & $E$ & $\mathrm{R}$ & $\mathrm{N}$ \\
\hline Lemus, et al. & MTT/7-10 días & MP/4-6 sem & 20 & $\mathrm{R}$ & - & - & - & 100 & - \\
\hline \multirow[t]{2}{*}{$2004^{18}$} & RES/7-10 días & & & & - & - & - & 100 & - \\
\hline & MNR/7-14 días & & & & - & - & - & 100 & - \\
\hline Montoro, et al. & MTT/7-10 días & MP/4-6 sem & 100 & $I, S, E, R$ & 98,0 & 90,0 & 75,0 & 100,0 & - \\
\hline \multirow[t]{2}{*}{$2005^{19}$} & RES/7-10 días & & & & 98,0 & 91,0 & 64,0 & 99,0 & - \\
\hline & MNR/7-14 días & & & & 98,0 & 96,0 & 99,0 & 100,0 & - \\
\hline Yzquierdo, et al. & $\mathrm{EAF} / 48 \mathrm{~h}$ & MP/4-6 sem & 102 & $\mathrm{R}$ & - & - & - & 99,0 & - \\
\hline \multicolumn{10}{|l|}{$2006^{20}$} \\
\hline Lemus, et al. & MNR/7-14 días & MP/4-6 sem & 320 & $\mathrm{I}, \mathrm{S}, \mathrm{E}, \mathrm{R}$ & 98,4 & 98,4 & 99,1 & 99,1 & - \\
\hline \multicolumn{10}{|l|}{$2006^{21}$} \\
\hline Mirabal, et al. & MNR/7-14 días & EEW/4-10 días & 102 & $\mathrm{~N}$ & - & - & - & - & 97,05 \\
\hline $2010^{22}$ & & & & & & & & & \\
\hline
\end{tabular}

MTT: ensayo colorimétrico MTT, RES: ensayo colorimétrico con resazurina, MNR: método de la nitrato reductasa, EAF: ensayo de amplificación de fagos, MP: método de las proporciones, EEW: ensayo enzimático de Wayne, I: isoniacida, S: estreptomicina,

$\mathrm{E}$ : etambutol, $\mathrm{R}$ : rifampicina, $\mathrm{N}$ : nicotinamida, sem: semanas.

\section{Diagnóstico de micobacterias no tuberculosas}

Las infecciones producidas por micobacterias atípicas $u$ oportunistas han tenido un significativo aumento a nivel mundial en los últimos 20 años, sobre todo desde la aparición de la epidemia del sida. Este aumento ha condicionado un incremento paralelo en la investigación y conocimiento de estos microorganismos, que ha conllevado a una estandarización en los criterios diagnósticos y terapéuticos. Para la identificación de especies micobacterianas a partir de muestras clínicas se han establecido diferentes esquemas fenotípicos de identificación basados en la velocidad de crecimiento, producción de pigmento y realización de una batería de pruebas bioquímicas que resultan laboriosas y demoran de 3 a 6 semanas en obtener los resultados. Por tal motivo, para la identificación de especies micobacterianas se han aplicado métodos cromatográficos basados en el análisis lipídico. ${ }^{26}$

Recientemente, el LNR-TB ha incorporado una nueva técnica para la identificación de las micobacterias basada en la amplificación por reacción en cadena de la polimerasa de un fragmento del gen de la proteína de estrés térmico de 65kDa. Este fragmento, se encuentra altamente conservado dentro del género Mycobacterium y la identificación a nivel de especie se puede realizar gracias al

http://scielo.sld.cu 
empleo de enzimas de restricción (BstEII y HaeIII), que digieren el material genético amplificado en fragmentos que son de tamaño conocido y específico para cada especie. Es un método sencillo y rápido pero requiere de determinados recursos y entrenamiento del personal que lo realiza. Su aplicación puede ser de gran utilidad para el diagnóstico de especies micobacterianas con características fenotípicas y bioquímicas similares. ${ }^{27}$

\section{Caracterización genotípica}

Desde finales del siglo pasado, la caracterización genotípica de aislamientos clínicos de $M$. tuberculosis, ha revolucionado el conocimiento de la transmisión de la TB brindándole una nueva dimensión a la epidemiología convencional y ha tenido un gran impacto en el control de la enfermedad a nivel mundial. Las técnicas de genotipificación existentes han permitido definir o refutar posibles errores de laboratorio y casos de reactivación latente o reinfección erógena, detectar y estudiar brotes nosocomiales identificando las cadenas de transmisión y los casos de infección secundaria. ${ }^{28}$ También se han aplicado a estudios genético-poblacionales en donde se ha determinado el porcentaje de transmisión reciente y los factores de riesgo asociados a ella encontrándose que este porcentaje ha sido mayor que el estimado anteriormente con herramientas epidemiológicas convencionales. Adicionalmente, las técnicas genotípicas han permitido conocer la estructura poblacional clonal de M. tuberculosis caracterizada por diferencias en la distribución geográfica, inmunogenicidad y la asociación con cepas MDR. ${ }^{29}$

La técnica de referencia para realizar la genotipificación de cepas de M. tuberculosis es el análisis de la longitud de los fragmentos de restricción (RFLP), con la secuencia de inserción IS6110 como molécula diana. El RFLP-IS6110 se basa en el análisis de la "huella dactilar" del ADN de diferentes aislamientos clínicos en cuanto al número de copias y posición en el genoma del elemento repetitivo IS6110.30,31

En Cuba, el RFLP-IS6110 se ha empleado como herramienta epidemiológica desde la década de los 90 . Se han realizado dos investigaciones genético-poblacionales en Ciudad de La Habana (actualmente La Habana) y en Cuba, en donde se encontró más del $45 \%$ de agrupamiento entre las cepas estudiadas, lo que demostró que en el periodo estudiado ocurrió un porcentaje elevado de transmisión reciente. ${ }^{32,33} \mathrm{El}$ análisis molecular de dos brotes nosocomiales ocurridos en un hospital y entre personas viviendo con el VIH/sida demostraron la importancia de la detección precoz de la enfermedad para evitar la diseminación de cepas de M. tuberculosis. ${ }^{34,35}$ Además, se pudo efectuar la confirmación molecular de un evento de contaminación cruzada ocurrida en una unidad de salud, ${ }^{36}$ y la caracterización genética de cepas resistentes a las drogas antituberculosas.

A pesar de ser el RFLP-IS6110 una técnica con elevado poder de discriminación y de gran aplicabilidad, tiene varias limitaciones, entre las más importantes están, que se necesita una gran cantidad de ADN purificado, es técnicamente demandante y de intensa labor, requiere gran cantidad de tiempo, tiene bajo poder discriminatorio en cepas con menos de seis copias de IS6110 y baja reproducibilidad entre laboratorios. ${ }^{28}$

Por este motivo, se han diseñado más de una decena de herramientas de caracterización genotípica alternativas (basadas fundamentalmente en la reacción en cadena de la polimerasa), sin embargo, la inmensa mayoría no ha pasado los controles internacionales por presentar problemas de reproducibilidad, confiabilidad o baja resolución. ${ }^{37}$ Las dos técnicas que más se emplean en la actualidad son: la tipificación con oligonucleótidos espaciadores (Spoligotyping, abreviatura en 
inglés $)^{38}$ y la tipificación con unidades repetitivas interespaciadas de micobacteriasrepeticiones en tándem de número variable (MIRU-VNTR, siglas en inglés). ${ }^{39}$

Spoligotyping se basa en la amplificación de la región aledaña a los elementos repetitivos directos (DR, en inglés) y una posterior hibridación diferencial de los productos amplificados con oligonucleótidos unidos a una membrana y que son complementarios a las regiones espaciadoras variables localizadas entre los DR. ${ }^{38}$ Con esta técnica se simplifica la comparación visual y se logra una excelente reproducibilidad $(100 \%)$, pero el poder de discriminación es menor que el del RFLP-IS6110 cuando se analizan cepas con alto número de copias del elemento IS6110. En el caso de cepas con bajo número de copias de dicho elemento, la diferenciación entre las mismas se logra con excelentes resultados. ${ }^{37,38}$ Otra ventaja adicional es la existencia de una gigantesca base de datos de patrones de Spoligotyping que se encuentra en el Instituto Pasteur de Guadalupe y que reúne a más de 75000 cepas procedente de 160 países. ${ }^{40}$ Debido a su simpleza, reproducibilidad y fácil comparación intra e interlaboratorio, es la técnica genotípica (fundamentalmente en estudios filogenéticos) que más se ha usado en la caracterización de M. tuberculosis. ${ }^{28,29}$

En cambio, la tipificación MIRU-VNTR se basa en la amplificación de múltiples sitios repetidos ( 15 o 24 loci), presentes en forma dispersa e interespaciada en el genoma micobacteriano, usa cebadores específicos para las regiones flanqueantes de cada sitio y la determinación de las tallas de los productos amplificados, las cuales reflejan el número de repeticiones en tándem presentes en cada sitio. La variabilidad en el número de repeticiones que exhibe cada cepa según el sitio amplificado, permite realizar su tipificación.

La tipificación MIRU-VNTR es más rápida y menos laboriosa que el RFLP-IS6110, se aplica a bajas concentraciones de ADN extraídas de cultivos mico bacterianos jóvenes y a formatos de alto alcance. Los resultados se expresan como códigos numéricos (basado en el número de secuencias repetitivas llamadas regiones polimórficas micro o minisatélites), y son también fácilmente comparables entre laboratorios. ${ }^{39}$ Un número importante de estudios demuestran que la tipificación por MIRU-VNTR es un método reproducible, confiable, que ofrece un nivel de discriminación comparable con la tipificación por RFLP-IS6110.39,40 Desde 2006, se ha propuesto emplear un subgrupo de 15 loci MIRU-VNTR altamente discriminatorios para investigaciones epidemiológicas de primera línea, mientras que se recomienda utilizar el grupo de 24 loci para estudios filogenéticos. ${ }^{39}$

En Cuba, se introdujo la técnica de tipificación por MIRU-VNTR-15 loci en 2009 y con ellos se genotipificaron 12 cepas MDR aisladas entre 2007 y 2009. La mayoría de ellas mostró patrones diferentes, sin embargo, tres de ellas tuvieron el mismo patrón genético. Con este trabajo se demostró la utilidad de la técnica para diferenciar cepas de TB y se creó una base de datos de patrones genotípicos de estas cepas MDR que ayudará en el futuro a rastrear eventos de transmisión de TB-MDR y detectar posibles casos "importados". ${ }^{41}$ Recientemente, se concluyó el primer estudio genético-poblacional realizado en Cuba con la tipificación por MIRUVNTR (15 o 24 loci). En este se analizaron 80 cepas de $M$. tuberculosis procedentes de La Habana aisladas en 2009. Se encontró un porcentaje elevado de cepas agrupadas $(53,75 \%)$, lo que refleja que en esta provincia debió ocurrir más transmisión reciente que lo que se sospechaba. ${ }^{42}$ En estos momentos se trabaja en la implementación de esta tecnología en la caracterización genotípica de la mayoría de las cepas recibidas en el LNR-TB procedentes de todo el país. 
El empleo de las técnicas de genotipificación de manera sistemática y mayoritaria, ayudará en la detección más temprana y oportuna de las fuentes de infección en instituciones cerradas y en la comunidad.

\section{Estudios taxonómicos}

Desde 1970, el LNR-TB aborda estudios sistemáticos de algunas micobacterias de difícil ubicación taxonómica. Es así que en 1971, Valdivia y otros registran el aislamiento de cepas de $M$. habana a partir de esputos de pacientes que presentaban un cuadro respiratorio indistinguible clínicamente de una TB pulmonar. ${ }^{43} \mathrm{Hasta}$ la fecha se han realizado diferentes estudios para diferenciar $M$. habana y $M$. simiae. Recientemente se han aplicado a estas dos especies micobacterianas diferentes técnicas cromatográficas y analíticas, lo que ha permitido confirmar diferencias muy significativas respecto a la composición de lípidos presentes en su pared, principalmente en las fracciones de ácidos micólicos y glicopeptidolípidos. ${ }^{44-47}$

Otro estudio en 16 cepas cromogénicas de crecimiento rápido aisladas de tejido animal permite registrar 3 nuevas especies: $M$. taurus, $M$. armentum y $M$. perpallidum. ${ }^{48}$ Asímismo en un estudio realizado en 1979 , se aisla y registra una nueva especie micobacteriana de crecimiento lento, a la que se denominó $M$. cubense. ${ }^{49}$

\section{Búsqueda de un inmunógeno protector contra la TB}

La especie $M$. habana ha despertado gran interés como inmunógeno protector contra la lepra y la TB. En un estudio de vacunación-protección realizado para evaluar 19 cepas de diferentes especies de micobacterias, los mejores valores de sobrevida corresponden a los ratones vacunados con la cepa M. habana TMC-5135. ${ }^{50}$ Atendiendo a estos resultados, en 1989 un reporte científico del TDR/OMS destaca el potencial de esta cepa como candidato vacunal frente a la TB. ${ }^{51}$

Entre los requisitos imprescindibles que se deben tener en cuenta durante la búsqueda de nuevos candidatos basados en el uso de vacunas vivas, se encuentran la virulencia de la cepa, su carácter inmunogénico y su capacidad para conferir protección contra la TB. En relación con estos, el LNR-TB ha llevado a cabo experimentos que permiten establecer el bajo poder de virulencia de las cepas de M. habana, lo que se demuestra en la esterilización de los tejidos de los animales infectados con esta cepa, en un modelo experimental murino de tuberculosis pulmonar progresiva. ${ }^{52}$ Los altos niveles de IFN- $\gamma$ obtenidos a partir de los tejidos de los ratones vacunados y estimulados in vitro con los principales antígenos de $M$. tuberculosis, indican su inmunogenicidad en este modelo. El reto con cepas virulentas de $M$ tuberculosis ( $\mathrm{H}_{37} \mathrm{Rv}$ y genotipo Beijing) en ratones inmunizados previamente con $M$. habana demuestra un mayor porcentaje de sobrevida para aquellos ratones vacunados con $M$. habana en comparación con el grupo control vacunado con BCG, estas diferencias son estadísticamente significativas, hecho que le atribuye poder protector a M. habana contra la TB. ${ }^{53}$

A pesar de que la mayoría de los estudios de inmunogenicidad realizados se enfocan hacia el empleo de cepas vivas de M. habana, se demuestra la existencia de mecanismos inmunológicos generados en respuesta a sus proteínas de secreción. ${ }^{54}$ Contar en el LNR-TB con una colección de aproximadamente 30 cepas de $M$. habana unido a los resultados obtenidos hasta el momento, reclaman futuras investigaciones en este campo, las cuáles abarcarán desde el uso, como 
inmunógeno, de proteínas secretadas de $M$. habana hasta los estudios de protección frente a otras entidades como la lepra.

\section{DESARROLLO ACTUAL Y PERSPECTIVO}

En el presente, el LNR-TB trabaja en el fortalecimiento de la red de diagnóstico de TB, para ello cuenta con el apoyo financiero de un proyecto del Fondo Mundial de lucha contra el sida, la tuberculosis y la malaria. Entre las tareas que se han ejecutado para lograr dichos objetivos se encuentran: mejorar la infraestructura de la red de diagnóstico y optimizar el diagnóstico por técnicas convencionales e implementar nuevas herramientas diagnósticas, perfeccionar la vigilancia de la drogoresistencia, detectar patrones de transmisión de la TB utilizando métodos moleculares, fortalecer la capacidad de los recursos humanos (talleres de capacitación) así como monitorear y evaluar la red de laboratorios (visitas de control y supervisión).

La ejecución y el cumplimiento de los objetivos planteados en dicho proyecto, apoyan al PNCT para lograr la eliminación de la TB como problema de salud en Cuba.

\section{Agradecimientos}

Agradecemos la colaboración de los técnicos Miguel Echemendía Font, Misleidis Sardiña Aragón y Grechen García León.

\section{REFERENCIAS BIBLIOGRÁFICAS}

1. Organización Panamericana de la Salud. Manual para el diagnóstico Bacteriológico de la Tuberculosis. Normas y Guía Técnica. Parte I Baciloscopía. Washington, D.C.: OPS; 2008.

2. Valdivia JA, Jiménez CA, Ferrá C, Mederos L, Echemendía M, Mazón D. El Laboratorio Nacional de Referencia de Mycobacteria y Tuberculosis dentro de la lucha antituberculosa en Cuba. Rev Cubana Med Trop. 1988;40:51-66.

3. Marrero A, Carreras L, Valdivia JA, Montoro E, González E, Torres R, et al. Programa Nacional de Control de la Tuberculosis. Manual de Normas y Procedimientos. La Habana: Editorial Ciencias Médicas; 1999.

4. Martínez MR, García G, Sardiña M, Díaz M, Columbie V, Pequero R, et al. Introducción de nuevos métodos de control de calidad de baciloscopia de esputo BAAR, en el Laboratorio Nacional de Referencia del Instituto de Medicina Tropical "Pedro Kourí". Rev Cubana Med Trop [Internet]. 2007 [citado 30 Nov 2011]. Disponible en: http://scielo.sld.cu/scielo.php?script=sci arttext\&pid=S0375$\underline{07602007000300013 \& \operatorname{lng}=\mathrm{es}}$

5. Martínez MR, García G, Sardiña M, Díaz M, Columbie V, Pequero R, et al. Evaluación de nuevos métodos para el control de calidad de la baciloscopia de tuberculosis en Cuba. Rev Inst Nal Enf Resp Mex. 2008;21:99-106. 
6. Organización Panamericana de la Salud. Manual para el diagnóstico Bacteriológico de la Tuberculosis. Normas y Guía Técnica. Parte II Cultivo. Washington, D.C.: OPS; 2008.

7. Sorlozano A, Soria I, Roman J, Huertas P, Soto MJ, Piedrola G, et al. Comparative evaluation of three culture methods for the isolation of mycobacteria from clinical samples. J Microbiol Biotechnol. 2009;19:1259-64.

8. Werngren J, Klintz L, Hoffner SE. Evaluation of a novel kit for use with the BacT/ALERT 3D System for drug susceptibility testing of Mycobacterium tuberculosis. J Clin Microbiol. 2006;44:21302.

9. Piersimoni C, Scarparo C, Callegaro A, Passerine Tosi C, Nista D, Bornigia S, et al. Comparison of MB/BacT ALERT 3D System with Radiometric BACTEC System and Lowenstein-Jensen Medium for recovery and identification of Mycobacteria from clinical specimens: a multicenter study. J Clin Microbiol. 2001;39:6517.

10. Marzouk M, Kahla IB, Hannachi N, Ferjeni A, Ben Salma W, Ghezal S, et al. Evaluation of an immunochromatographic assay for rapid identification of Mycobacterium tuberculosis complex in clinical isolates. Diag Microbiol Infect Dis. 2011;69:3969.

11. Gaillarda T, Fabreb M, Martinauda C, Vongb R, Brisoua P, Solerb Ch. Assessment of the SD Bioline Ag MPT64 Rapid ${ }^{\mathrm{TM}}$ and the MGIT ${ }^{\mathrm{TM}}$ TBC identification tests for the diagnosis of tuberculosis. Diag Microbiol Infect Dis. 2011;70:1546.

12. Montoro E. Multiple drug resistance: a threat for tuberculosis control. Pan Am J Public Health. 2004;16:68-73.

13. Migliori GB, Matteelli A, Cirillo D, Pai M. Diagnosis of multidrug-resistant tuberculosis and extensively drug-resistant tuberculosis: Current standards and challenges. Can J Infect Dis Med Microbiol. 2008;19:169-72.

14. Valdivia JA, Suarez R. Incidencia y prevalencia de cepas drogorresistentes en el Hospital antituberculoso "Julio Trigo"de La Habana. Bol Hig Epidemiol. 1968;6:247-51.

15. Valdivia JA, Jiménez $C$, Rodríguez $R$, Mederos $L$, Echemendía $M$, Valdés $L$, et al. Estudio cooperativo de la resistencia a las drogas antibacilares en cepas de $M$. tuberculosis aisladas en pacientes tuberculosos. (Informe preliminar). Rev Cubana Med Trop. 1982;34:119-25.

16. Pablos Méndez A, Raviglione MC, Laszlo A, Binkin N, Rieder $H L$, Bustreo $F$, et al. For the World Health Organization-International Union against Tuberculosis and Lung Disease Working Group on Anti-Tuberculosis Drug Resistance Surveillance. Global surveillance for antituberculosis-drug resistance, 1994-1997. N Engl J Med. $1998 ; 338: 1641-9$.

17. World Health Organization-International Union against Tuberculosis and Lung Disease Global Project and Anti-Tuberculosis Drug Resistance Surveillance. Antituberculosis drug resistance in the world. Report No. 2 Prevalence and trends. Geneva: WHO; 2000.

18. World Health Organization-International Union against Tuberculosis and Lung Disease. Global Project and Anti-Tuberculosis Drug Resistance Surveillance, 19992002. Third Global Project, 2004. Anti-tuberculosis drug resistance in the world. Geneva: WHO; 2000. 
19. World Health Organization-International Union against Tuberculosis and Lung Disease. Global Project and Anti-Tuberculosis Drug Resistance Surveillance, Fourth Global Project, 2008. Anti-tuberculosis drug resistance in the world. Geneva: WHO; 2008.

20. Montoro E, Lemus D, Echemendía M, Armas L, González Ochoa E, Llanes MJ, et al. Drug-resistant tuberculosis in Cuba. Results of the three global projects. Tuberculosis. 2006;86:319-23.

21. Lemus D, Martín A, Montoro E, Portaels F, Palomino JC. Rapid alternative methods for detection of rifampicin resistance in Mycobacterium tuberculosis. J Antimicob Chemother. 2004;54:130-3.

22. Montoro E, Lemus D, Echemendía M, Martin A, Portaels F, Palomino JC. Comparative evaluation of the nitrate reduction assay, the MTT test and the resazurin microtitre assay for drug susceptibility testing of clinical isolates of Mycobacterium tuberculosis. J Antimicrob Chemother. 2005;55:500-5.

23. Yzquierdo SL, Lemus $D$, Echemendía M, Montoro E, McNerney R, Martin A, et al. Evaluation of phage assay for rapid phenotypic detection of rifampicin resistance in Mycobacterium tuberculosis. Ann Clin Microbiol Antimicrob. 2006;5:11.

24. Lemus D, Montoro E, Echemendía M, Martin A, Portaels F, Palomino JC. Nitrate Reductase Assay for detection of drug resistance in Mycobacterium tuberculosis: simple and inexpensive method for low resources laboratories. J Med Microbiol. 2006;55:861-3.

25. Mirabal N, Yzquierdo S, Lemus D, Madruga M, Milian Y, Echemendía M, et al. Evaluation of colorimetric methods using nicotinamide for rapid detection of pyrazinamide resistance in Mycobacterium tuberculosis. J Clin Microbiol. 2010;48:2729-33.

26. Leao SC, Martin A, Mejia GI, Palomino JC, Robledo J, Telles MA, Portaels F. Practical handbook for the phenotypic and genotypic identification of Mycobacteria. Brugge, Belgium: Vanden Broelle; 2004.

27. Yzquierdo SL, Mederos LM, Díaz A, Echemendía M, Montoro E. Aplicación de RPC-PLFR en el diagnóstico de micobacterias no tuberculosas. Rev Chil Infect. 2007;24:391-6.

28. Van Soolingen D. Molecular epidemiology of tuberculosis and other mycobacterial infections: main methodologies and achievements. J Inter Med. 2001;249:1-26.

29. Behr MA, Mostowy S. Molecular tools for typing and branding the tubercle bacillus. Curr Mol Med. 2007;7:309-17.

30. Van Embden JD, Cave MD, Crawford JT. Strain identification of Mycobacterium tuberculosis by DNA fingerprinting: recommendations for a standardized methodology. J Clin Microbiol. 1993;31:406-9.

31. Kremer K, Arnold C, Cataldi A, Gutiérrez MC, Haas WH, Panaiotov S, et al. Discriminatory power and reproducibility of novel DNA typing methods for Mycobacterium tuberculosis complex strains. J Clin Microbiol. 2005;43:5628-38.

32. Díaz R, de Haas P, Gómez RI, Marrero A, Cabanas M, Valdivia JA, et al. Molecular Epidemiology of Tuberculosis in Cuba, 1994-1995; Comparison of IS6110-RFLP and Spoligotyping. Int J Tuberc Lung Dis. 1998;2:743-50.

http://scielo.sld.cu 
33. Díaz R, Gómez RI, Restrepo E, Rumbaut R, Valdivia JA, Soolingen D. Transmission of tuberculosis in Havana, Cuba: a molecular epidemiological study by IS6110 Restriction fragment length polymorphism typing. Mem Inst Oswaldo Cruz. 2001;96:437-43.

34. Díaz R, Montoro E, González R, Echemendía M, Valdivia JA. Analysis of Mycobacterium tuberculosis strains isolated during an outbreak of HIV-infected patients by DNA fingerprinting. Am J Trop Med Hyg. 1995,53:S155-6.

35. Díaz R, Gómez RI, García N, Valdivia JA, Soolingen D. Molecular epidemiology study on transmission of tuberculosis in a hospital for mentally handicapped patients in Havana, Cuba. J Hosp Infect. 2001;49:30-6.

36. Díaz R, Crespo FM, Herrera S, Sevy Court J, Marrero A, Soolingen D. Laboratory cross-contamination of Mycobacterium tuberculosis during preparation of smears. Internet J Third World Med. 2005;2.

37. Kremer K, van Soolingen D, Frothingham R, Hass WH, Hermans PWM, Martin C, et al. Comparison of methods based on different molecular epidemiological markers for typing of Mycobacterium tuberculosis complex strains: interlaboratory study of power of discrimination and reproducibility. J Clin Microbiol. 1999;37:2607-18.

38. Kamerbeek J, Kock A, van Agterveld M, van Soolingen D, Kuijper S, Burshoten A. Rapid detection and simultaneous strain differentiation of Mycobacterium tuberculosis for diagnosis and tuberculosis control. J Clin Microbiol. 1997;35:907-14.

39. Supply P, Allix C, Lesjean S, Cardoso Oelemann M, Rusgh Gerdes S, Willery E, et al. Proposal for standardization of optimized mycobacterial interspersed repetitive unit-variable-number tandem repeat typing of Mycobacterium tuberculosis. J Clin Microbiol. 2006;44:4498-510.

40. García de Viedma D, Mokrousov I, Rastogi N. Innovations in the molecular epidemiology of tuberculosis. Enferm Infec Microbiol Clin. 2011;29 (Suppl 1):8-13.

41. Diaz R, González Y, Goza R, Lemus D, Montoro E. Genotyping of multidrugresistant (MDR) Mycobacterium tuberculosis isolates from Cuba, 2000-2008. Int J Tuberc Lung Dis. 2009;13(12):S368.

42. Diaz R, Goza R, Montoro E. Variable number tandem repeat typing of Mycobacterium tuberculosis isolates from Havana, Cuba. Int J Tuberc Lung Dis. 2011,15(11):S278.

43. Valdivia JA, Suárez R, Echemendía M. Mycobacterium habana: probable nueva especie dentro de las micobacterias no clasificadas. Bol Hig Epidemiol. 1971;9:65-73.

44. Mederos LM, Valdivia JA, Valero Guillén PL. Analysis of lipids reveals differences between Mycobacterium habana and Mycobacterium simiae. Microbiology. $1998 ; 144: 1181-8$.

45. Mederos LM, Valdivia JA, Valero Guillén PL. Lipids of "Mycobacterium habana", a synonym of Mycobacterium simiae with vacontrol de la calidadine potencial. Tuberculosis. 2006;86:324-9.

46. Mederos LM, Valdivia JA, Valero-Guillén PL. Analysis of the structure of mycolic acids of Mycobacterium simiae reveals a particular composition of alpha-mycolates in strain 'M. habana' TMC 5135, considered as immunogenic in tuberculosis and leprosy. Microbiology. 2007;153:4159-65. 
47. Mederos LM, Valdivia JA, Valero Guillén PL. New variants of polar glycopeptidolipids detected in Mycobacterium simiae including 'M. habana' strains, as evidence by electrospray ionization-ion trap-mass spectrometry. J Appl Microbiol. 2008;105:602-14.

48. Valdivia JA, Ferrá C, Echemendía M. Estudio de 16 cepas de Micobacterias cromogénicas y de crecimiento rápido. Rev Cubana Med Trop. 1975;27:213-23.

49. Valdivia JA, Echemendía M, Inerarity M. Mycobacterium cubense: nueva especie pigmentada y de crecimiento lento. Rev Cubana Med Trop. 1979;31:85-8.

50. Gupta HP, Singh NB, Mathur IS, Gupta SK. 'Mycobacterium habana' a new immunogenic strain in experimental tuberculosis of mice. Ind J Exp Biol. $1979 ; 17: 1190$.

51. World Health Organization. Report of the 3th Anual Immnunology (IMLEP) and Chemotherapy (THELEP) of Leprosy, Molecular Biology, Subcommitte (BIOLEP). Geneva: WHO; 1989.

52. Hernández Pando R, Orozco H, Arriaga K, Sampieri A, Larriva Sahd J, Madrid Marina V. Analysis of the local kinetics and localization of interleukin-1 alpha, tumour necrosis factor- $\alpha$ and transforming growth factor- $\beta$, during the course of experimental pulmonary tuberculosis. Immunology. 1997;90:60717.

53. Valdés, I, Echemendía M, Mederos L, Valdivia JA, Montoro E. Aspectos relevantes del uso de Mycobacterium habana como candidato vacunal contra la tuberculosis. Vaccimonitor. 2011;20(3):34-9.

54. Divya JM, Garg SK, Singh NB. Mechanisms involved in protective immune response generated by secretory proteins of Mycobacterium habana against experimental tuberculosis. Scand J Immunol. 2000;51:502-10.

Recibido: 22 de enero de 2010.

Aprobado: 5 de febrero de 2010.

Ernesto Montoro Cardoso. Instituto de Medicina Tropical "Pedro Kourí". Autopista Novia del Mediodía Km 6 1/2, La Lisa. La Habana, Cuba.

Correo electrónico: EMontoro@ipk.sld.cu 